ISSN 1981-416X

Licenciado sob uma Licença Creative Commons

\title{
Traços de personalidade e persistência discente em cursos superiores na modalidade a distância
}

\author{
Traits of personality and persistence in distance \\ education courses
}

\section{Trazos de personalidad y persistencia discente en cursos superiores en el educación a distancia}

\author{
Teresa Cristina Janes Carneiro ${ }^{[a]}$, Marcello Ferreira ${ }^{[b]}$, Ralf Luís de Moura ${ }^{[c]^{*}}$ \\ [a] Universidade Federal do Espírito Santo (UFES), Vitória, ES, Brasil \\ [b] Universidade de Brasília (UnB), Brasília, DF, Brasil \\ [c] Centro Universitário FAESA, Vitória, ES, Brasil
}

\section{Resumo}

Este artigo ${ }^{1}$ visa comparar características dos traços de personalidade dos discentes que persistem em cursos EaD. Os levantamentos foram realizados em licenciaturas e especializações oferecidas pela Universidade Federal do Espírito Santo (UFES) no âmbito do Sistema Universidade Aberta do Brasil (UAB). Para análise dos dados, utilizou-se o conceito de traços de personalidade proposto no Modelo Metateórico de Motivação e

${ }^{1}$ Este artigo deriva de parte de projeto submetido à Chamada Universal MCT/CNPq no 14/2014.

*TCJC: Doutora em Administração, e-mail: teresa.carneiro@ufes.br MF: Doutor em Educação em Ciências, e-mail: marcellof@unb.br RLM: Mestre em Engenharia elétrica, e-mail: ralf.luis@faesa.br 
Personalidade - 3M (MOWEN, 2000) e a análise de variância (ANOVA) como procedimento estatístico. Os resultados mostram que estudantes do gênero feminino são significativamente mais amáveis, conscienciosas, autoeficazes no aprendizado, têm menos necessidade de excitação, mais necessidade de aprendizado, possuem mais consciência de valor, estão mais satisfeitas com os cursos e são mais persistentes. Discentes casados possuem maior consciência de valor e menor necessidade de excitação. Quanto mais alta a renda, maior a necessidade de recursos materiais, maior a orientação para tarefas e menor a introversão. Em cursos de especialização, são mais abertos a experiências, mais conscienciosos, possuem mais necessidade de recursos materiais e corporais, são mais interativos, aceitam mais a tecnologia e possuem maior consciência de valor. Em cursos das áreas de Humanidades e Ciências Sociais, são mais amáveis, abertos a experiências, conscienciosos e possuem maior necessidade de recursos materiais e maior consciência de valor do que os discentes das áreas de Ciências Exatas e Biológicas. A partir desses achados, espera-se contribuir com políticas e ações universitárias que promovam a persistência discente, sobretudo em cursos EaD.

Palavras-chave: Persistência discente. Ensino superior. Educação a distância. Traços de personalidade. Modelo 3M.

\section{Abstract}

This article aims to compare the characteristics of the personality traits of students who persist in Distance Education courses. The surveys were carried out in bachelors and postgraduate courses offered by the Federal University of Espírito Santo (UFES) under the Open University System of Brazil (UAB). In order to analyze the data, we used the concept of personality traits proposed in the Meta-Motivational and Personality Model - 3M (MOWEN, 2000) and the analysis of variance (ANOVA) as a statistical procedure. The results show that female students are significantly kinder, more conscientious, self-efficacious in learning, less need for excitement, more need for learning, more aware of the value, more satisfied with courses, and more persistent. Married couples have a greater awareness of value and less need for excitement. The higher the income, the greater the need for material resources, the greater the orientation to tasks and the less the introversion. In 
postgraduate courses, they are more open to experiences, more conscientious, more in need of material and bodily resources, more interactive, more accepting of technology, and more aware of their value. In Humanities and Social Sciences, they are more kind, open to experiences, conscientious and have greater need of material resources and greater awareness of value than the students of the areas of Exact and Biological Sciences. Based on these findings, it is hoped to contribute to university policies and actions that promote student persistence, especially in EaD courses.

Keywords: Student persistence. Higher education. Distance education. Personality traits. 3M Model.

\section{Resumen}

Este artículo busca comparar características de los rasgos de personalidad de los discursos que persisten en cursos en el educación a distancia. Los levantamientos se realizaron en licenciaturas y especializaciones ofrecidas por la Universidad Federal de Espírito Santo (UFES) en el ámbito del Sistema Universidad Abierta de Brasil (UAB). Para el análisis de los datos, se utilizó el concepto de rasgos de personalidad propuesto en el Modelo Metateórico de Motivación y Personalidad - 3M (MOWEN, 2000) y el análisis de varianza (ANOVA) como procedimiento estadístico. Los resultados muestran que los estudiantes del género femenino son significativamente más amables, concienzudos, autoeficaces en el aprendizaje, tienen menos necesidad de excitación, más necesidad de aprendizaje, poseen más conciencia de valor, están más satisfechas con los cursos y son más persistentes. Los discursos casados poseen mayor conciencia de valor y menor necesidad de excitación. Cuanto más alta es la renta, mayor es la necesidad de recursos materiales, mayor es la orientación para tareas y menor la introversión. En cursos de especialización, son más abiertos a experiencias, más concienzudos, poseen más necesidad de recursos materiales y corporales, son más interactivos, aceptan más la tecnología y tienen mayor conciencia de valor. En cursos de las áreas de Humanidades y Ciencias Sociales, son más amados, abiertos a experiencias, concienzudos y poseen mayor necesidad de recursos materiales y mayor conciencia de valor que los discentes de las áreas de Ciencias Exactas y Biológicas. 
A partir de esos hallazgos, se espera contribuir con políticas y acciones universitarias que promuevan la persistencia discente, sobre todo en cursos EaD.

Palabras clave: Persistencia estudiantil. Educación superior. Educación a distancia. Rasgos de personalidade. Modelo $3 \mathrm{M}$.

\section{Introdução}

Transcorridas mais de duas décadas do reconhecimento como modalidade educacional e mais de uma década da sua regulação, a Educação a Distância (EaD) tem transformado a formação superior no Brasil de forma quantitativa (pelas proporções de matrículas e por sua capilaridade) e qualitativa (pelo inevitável fomento ao uso de tecnologias). Ao mesmo passo, incrementou e deu escalas variadas a desafios já enfrentados pelas instituições de ensino superior que ofertam cursos nessa modalidade, em especial a evasão discente.

Estudo feito pelo Instituto Lobo para o Desenvolvimento da Educação, da Ciência e da Tecnologia, com base nos dados do Censo do Ensino Superior, mostra que apenas $49 \%$ dos estudantes desse nível de ensino se titularam após quatro anos de curso. As perdas financeiras com a evasão no ensino superior podem chegar a 27\% (SILVA FILHO, 2017). Estudos realizados em 2013 na Universidade Federal do Espírito Santo (UFES) apontam que apenas $48 \%$ dos estudantes que deveriam se formar em 2012 concluíram seus cursos, confirmando os resultados do Instituto Lobo (PEREIRA, 2013; SALES JUNIOR, 2013).

Outro dado importante, relacionado à eficiência do sistema de ensino superior, é a Taxa de Titulação, que mede o percentual de alunos que se forma em relação ao número de ingressantes nos anos de ingresso correspondentes. No caso brasileiro, dados do Censo do Ensino Superior mostram que a Taxa de Titulação, relacionando os formados de 2015 aos ingressantes de 2011 para os cursos de licenciatura $\mathrm{EaD}$, foi de 51\%, significando que 
apenas aproximadamente metade dos discentes se formaram no período previsto (SILVA FILHO, 2017). A retenção discente, entendida como a não integralização do curso no período recomendado no projeto pedagógico, é um dos fatores que podem levar à evasão discente (PEREIRA et al., 2016).

Segundo Tinto (2012), alunos de renda elevada têm três vezes mais chances de concluir o ensino superior, em comparação àqueles de baixa renda. Assim, quando se amplia o acesso dos estudantes de menor renda ao ensino superior, proporcionalmente em relação a aqueles de mais alta renda, a evasão ganha nova proporção. Ainda segundo Tinto (2012), a despeito da intenção de várias nações de ampliar o acesso de estudantes de baixa renda à universidade, buscando reduzir desigualdades sociais, ainda não é possível ver esse esforço espelhado, nas mesmas proporções, nos índices de conclusão dos cursos superiores.

Esses resultados não podem ser imputados à falta de esforços. Governos de vários países têm investido grandes somas de recursos em programas de apoio e assistência estudantil, visando reduzir a evasão no ensino superior ${ }^{2}$, grande parte deles voltados especificamente aos estudantes de baixa renda (HESS et al., 2009; MORTENSEN, 2012).

Apesar disso, pesquisas que buscam compreender o fenômeno da evasão discente não têm tido sucesso em apontar soluções para o desenvolvimento e implantação de programas que reduzam a desistência (SALLES JUNIOR., 2013; ALENCAR, 2014; PETRIS, 2014). Segundo Tinto (2012), isso se deve ao fato de essas pesquisas considerarem que o fenômeno da persistência é o inverso do fenômeno da evasão. Supõem que, ao se compreender a evasão discente, automaticamente pelo seu inverso, compreende-se o comportamento de persistência ou de não desistência. Apesar dos fenômenos estarem relacionados, compreender as razões que levam à evasão não necessariamente propicia o desenvolvimento de ações que ajudem os estudantes a persistirem. A persistência parece estar associada a questões singulares do indivíduo, tais como sua personalidade e motivação.

${ }^{2}$ A esse respeito, ver estatísticas em Tinto (2012), por exemplo. 
Soma-se a isso o fato de que estudantes tradicionais, que se dedicam em tempo integral ao ensino superior, após concluírem o ensino médio representam menos da metade dos estudantes das universidades. A maioria deles trabalha enquanto cursa o nível superior, especialmente aqueles de baixa renda e mais velhos, que não tiveram oportunidade de cursar o ensino superior logo após completar o ensino médio. Esses estudantes, denominados não tradicionais, são a maioria nos cursos $\mathrm{EaD}$, o que contribui para o agravamento do problema do baixo índice de conclusão dos cursos nessa modalidade educacional (METZNER; BEAN, 1987).

Como um traço psicológico ou característica de personalidade, a persistência pode ser definida como: 1) perseverança ou repetição de uma ação, procedimento ou atividade apesar da cessação do estímulo que a originou; 2) a qualidade ou estado de preservar um caminho de ação ou continuar um trabalho e completá-lo a despeito de forças contrárias ou esforço envolvido ${ }^{3}$. Assim, persistência é a capacidade de continuar com os esforços mesmo frente a desafios ou dificuldades. É o comportamento de ação contínua, apesar da presença de obstáculos (ROVAI, 2003).

A persistência na aprendizagem tem sido definida como o "estado em que alunos participam continuamente de seus programas educacionais e concluem seus cursos, créditos e graus para chegar a seus objetivos educacionais" (MÜLLER, 2008, p. 2). Lee (2003) identificou que a motivação do aluno é fator importante para a persistência discente; já Chung (2000) afirma que alunos persistentes em cursos EaD apresentam capacidade de motivação intrínseca e participam de forma mais ambiciosa do processo de aprendizagem.

Os adultos optam por participar de programas educacionais para atender às suas metas de aprendizagem ou crescimento profissional, enquanto as crianças, com menor autonomia, participam da escola por causa de requisitos de frequência obrigatória. Consequentemente, as taxas de persistência dos adultos são muitas vezes substancialmente mais

${ }^{3}$ Psychology Dictionary. What is persistence? Disponível em: <http://psychologydictionary.org/ persistence >. Acesso em: 20 out. 2018. 
baixas do que as de crianças, fortemente relacionadas com a capacidade dos programas educacionais em satisfazer às necessidades desses adultos (ROVAI, 2003).

Durante as últimas décadas, têm surgido vários modelos teóricos sobre persistência discente no ensino superior. As primeiras tentativas de explicar a persistência são baseadas em modelos psicológicos. Fishbein e Ajzen (1975) teorizam que a decisão do aluno de persistir é, em grande medida, baseada em comportamentos anteriores, atitudes e normas que direcionam o seu comportamento para a intenção do aprendizado.

Tipologias mais recentes, fundamentadas em modelos psicológicos, explicam a persistência levando em consideração o estudante, a instituição de ensino e suas variáveis ambientais, tais como a integração social dos alunos na vida no campus. Nessa linha, dois modelos importantes foram desenvolvidos por Tinto $(1975 ; 1987 ; 1993)$ e por Bean e Metzner (1985). Tinto $(1975 ; 1987 ; 1993)$ teorizou que os principais determinantes da persistência bem-sucedida podem ser divididos em: (i) fatores relacionados a experiências anteriores à entrada no curso/ (ii) características individuais dos estudantes; e (iii) fatores relacionados a experiências na universidade, durante a integralização do curso superior.

Ao analisar estudantes não tradicionais, Bean e Metzner (1985) identificaram quatro fatores que afetam a persistência: (i) variáveis acadêmicas - hábitos de estudo e disponibilidade de tempo para os estudos; (ii) variáveis individuais - idade, objetivos educacionais, etnia e resultados educacionais prévios; (iii) variáveis ambientais - finanças, horas de trabalho, responsabilidades familiares e estímulos exteriores; e (iv) resultados acadêmicos e psicológicos durante o curso. Essas variáveis, muitas das quais estão fora do controle da escola ou da universidade, podem induzir a desistência dos alunos, ao passo que pressionam seu tempo, seus recursos e sua sensação de bem-estar.

Metzner e Bean (1987) descobriram também que variáveis de integração não foram significativas para os estudantes não tradicionais. Para esses alunos, o que afeta a persistência é a percepção de utilidade da educação de nível superior na obtenção de emprego, satisfação pessoal 
e oportunidade de melhoria de vida. Para Henry e Smith (1993), os estudantes podem abandonar a universidade apesar do bom desempenho acadêmico caso percebam baixos níveis de utilidade do curso, não tenham satisfação ou se vivenciem altos níveis de estresse. Bean (1980) destaca, ainda, um aspecto importante para gestão das universidades ao afirmar que reter um aluno é fundamental para a capacidade de uma instituição realizar sua missão. A elevada taxa de abandono constitui-se uma falha da instituição para atingir o seu objetivo.

Seguindo a mesma linha de investigação sobre características dos estudantes, um desenvolvimento teórico mais recente (MOWEN, 2000; MOWEN; VOSS, 2008) tem possibilitado investigar construtos comportamentais associados a traços de personalidade em uma perspectiva hierárquica, integrando-os e possibilitando melhores índices de validade e confiabilidade. Neste trabalho, foram utilizados como referência os traços de personalidade sugeridos por Mowen (2000) que associa hierarquicamente traços básicos de personalidade a comportamentos que podem ser intersubjetivamente validados.

Sendo assim, o objetivo da presente pesquisa é comparar diferentes características quanto aos traços de personalidade de discentes que persistem em cursos superiores EaD. Consiste em analisar se o comportamento de persistência varia (e com quais correlações) em função dessas características.

\section{Referencial teórico}

Entende-se por traço de personalidade "qualquer construto intrapsíquico que pode ser mensurado de forma válida e confiável e que prevê diferenças individuais em sentimentos, pensamentos e comportamentos" (MOWEN, 2000, p. 2). O Modelo Metateórico de Motivação e Personalidade - 3M, proposto por Mowen (2000), é uma metateoria por fundamentar-se nos princípios da Teoria do Controle (CARVER; SCHEIRER, 1990), da Psicologia Evolucionária (BUSS, 1988), da 
Personalidade Hierárquica (PAUNONEN, 1998) e no Modelo dos Cinco Fatores de Personalidade (McCRAE; COSTA, 1997).

A estrutura hierárquica do Modelo 3M deriva do trabalho de Paunonen (1998) que sugere a organização da personalidade em quatro níveis hierárquicos, segundo níveis crescentes de abstração. O primeiro nível é o mais superficial, no qual se enquadram os comportamentos que podem ser facilmente observados (respostas específicas). O segundo nível reúne tendências comportamentais que podem ser observadas ao longo do tempo (respostas habituais). O terceiro nível congrega as preferências comportamentais (traços comportamentais) que antecedem as tendências comportamentais. O quarto e último nível contém fatores mais abstratos e abrangentes que antecedem os traços comportamentais. A partir dessa estratificação, Mowen (2000) propõe quatro níveis hierárquicos de traços de personalidade no modelo 3M: (i) traços elementares; (ii) traços compostos; (iii) traços situacionais; e (iv) traços superficiais. A hierarquia de personalidade proposta por Mowen, de traços mais abstratos a traços mais concretos, está apresentada de forma invertida em relação à perspectiva adotada por Paunonen (1998).

Os traços elementares são entendidos como construtos unidimensionais subjacentes às preferências e às predisposições individuais que se originam na herança genética e na história de aprendizagem na primeira infância do indivíduo, servindo de ampla referência a atitudes, sentimentos e comportamentos (MOWEN, 2000). Cinco dos traços elementares do modelo $3 \mathrm{M}$ originam-se do modelo de cinco fatores da personalidade (McCRAE; COSTA, 1997), a saber: abertura à experiência; conscienciosidade; introversão; amabilidade e instabilidade emocional. Da Psicologia Evolucionária (BUSS, 1988), somaram-se os traços associados a recursos sociais, cognitivos, materiais e corporais que os primatas necessitam para sobreviver. Por último, o Modelo 3M propõe a inclusão do traço de necessidade de excitação, a partir do trabalho de Mehrabian e Russel (1974 apud MOWEN, 2000) e Zuckerman (1979), totalizando os oito traços elementares apresentados no Quadro 1. 
Quadro 1 - Definições dos oito traços elementares do modelo 3M

\begin{tabular}{|l|l|}
\hline \multicolumn{1}{|c|}{ Traço } & \multicolumn{1}{c|}{ Definição } \\
\hline Abertura à Experiência & $\begin{array}{l}\text { A necessidade de achar soluções inovadoras, expressar ideias } \\
\text { originais e usar a imaginação ao realizar tarefas. }\end{array}$ \\
\hline Conscienciosidade & $\begin{array}{l}\text { A necessidade de ser organizado, ordeiro e eficiente ao realizar as } \\
\text { tarefas. }\end{array}$ \\
\hline Introversão & A tendência de revelar sentimentos de timidez e acanhamento. \\
\hline Amabilidade & A necessidade de expressar solidariedade e simpatia pelos outros. \\
\hline Instabilidade Emocional & $\begin{array}{l}\text { A tendência de reagir de forma emocional, expressando oscilações de } \\
\text { humor, e de ser temperamental. }\end{array}$ \\
\hline $\begin{array}{l}\text { Necessidade de Recursos } \\
\text { Materiais }\end{array}$ & A necessidade de obter e possuir bens materiais. \\
\hline Necessidade de Excitação & O desejo por estímulos e excitação. \\
\hline $\begin{array}{l}\text { Necessidades de } \\
\text { Recursos Corporais }\end{array}$ & A necessidade de manter e melhorar o corpo. \\
\hline
\end{tabular}

Fonte: Adaptado de Mowen (2000).

No segundo nível da hierarquia, encontram-se os traços compostos que resultam das predisposições primárias (traços elementares), influências culturais e história pessoal de aprendizagem. Segundo a concepção hierárquica do Modelo 3M, os níveis progressivamente se tornam menos abstratos e mais específicos. Inicialmente, Mowen (2000) propõe seis traços compostos, apresentados no Quadro 2.

\section{Quadro 2 - Definições dos seis traços compostos do Modelo 3M}

\begin{tabular}{|l|l|}
\hline \multicolumn{1}{|c|}{ Traço } & \multicolumn{1}{c|}{ Definição } \\
\hline Necessidade Aprendizado & $\begin{array}{l}\text { Tendência de se engajar em desafios intelectuais significativos e } \\
\text { apreciá-los. }\end{array}$ \\
\hline Orientação para Tarefas & $\begin{array}{l}\text { Disposição de definir um conjunto de atividades e atingir elevados } \\
\text { níveis de desempenho ao completar tarefas. }\end{array}$ \\
\hline Necessidade Atividades & Necessidade de gastar energia em atividades corporais e físicas. \\
\hline Necessidade de Diversão & $\begin{array}{l}\text { Tendência de realizar atividades hedonistas (diversão, fantasia, } \\
\text { excitação e estimulação sensorial) sem objetivos produtivos imediatos. }\end{array}$ \\
\hline Competitividade & $\begin{array}{l}\text { Propensão de participar em competições interpessoais e o desejo de } \\
\text { vencer e ser superior aos outros. }\end{array}$ \\
\hline Autoeficácia & $\begin{array}{l}\text { Capacidade intrínseca de organizar e executar ações requeridas de } \\
\text { acordo com os recursos pessoais percebidos. }\end{array}$ \\
\hline
\end{tabular}

Fonte: Adaptado de Mowen (2000). 
Os traços compostos utilizados nesta pesquisa são: autoeficácia (devido à necessidade de maior autonomia do estudante de cursos a distância), necessidade de aprendizado (por ser característico de quem procura um curso superior) e orientação para tarefas (pela característica de cursos a distância serem baseados em tarefas postadas em ambientes virtuais de aprendizagem com prazos sistematicamente estabelecidos).

Os traços situacionais, construtos de terceiro nível do Modelo $3 \mathrm{M}$, representam tendências de agir em relação a contextos gerais de comportamento. Resultam dos traços elementares e compostos, do contexto social, do tempo e da definição da atividade a ser realizada sendo, portanto, específicos para cada contexto. Como consequência, a variedade dos traços situacionais é naturalmente grande e é esperado que esses traços sejam altamente preditivos dos traços superficiais, em virtude de sua posição hierárquica muito próxima dos comportamentos concretos pesquisados. Os traços situacionais considerados mais relevantes a serem utilizados nessa pesquisa são: satisfação com o curso (LEVY, 2007; PITTENGER; DOERING, 2010; PARK; PERRY; EDWARDS, 2011; JOO; JOUNG; SIM, 2011; JOO; LIM; KIM, 2011); consciência de valor (MOWEN, 2000); aceitação da tecnologia — ou percepção de utilidade e facilidade de uso de tecnologias (HOLDER, 2007; JOO; LIM; KIM, 2011) e interação (SHIN, 2003; FINNEGAN; MORRIS; LEE, 2008; LEE; MORRIS; FINNEGAN, 2008; NISTOR; NEBAUER, 2010).

Satisfação com o curso é a capacidade de o estudante fazer associação positiva entre si e sua experiência educacional (SHIN, 2003). Segundo Rovai (2003), as taxas de persistência dos alunos não tradicionais, típicos de cursos $\mathrm{EaD}$, estão fortemente associadas à capacidade em satisfazer às necessidades destes, que podem chegar a abandonar a universidade, mesmo apresentando bom desempenho acadêmico, se tiverem baixos níveis de satisfação.

Consciência de valor, segundo Mowen (2000), é capacidade dos indivíduos de comparar custos e benefícios, investigar qualidade de 
produtos e serviços, barganhar e atuar de forma mais racional em relação a recursos já investidos, possivelmente mostrando maior tendência de abandonar projetos cujo retorno é ruim.

Aceitação ou propensão à tecnologia, segundo Mick e Fournier (1998), é a capacidade de um indivíduo de aceitar a tecnologia, que nem sempre é percebida de forma positiva pelos usuários. Ao mesmo tempo em que gera sentimentos positivos de controle, liberdade, novidade, competência, eficiência, satisfação, associação e engajamento, pode também provocar sentimentos negativos de confusão, dependência, obsolescência, incompetência, ineficiência, insatisfação, isolamento e desengajamento. Esses sentimentos negativos podem ensejar mecanismos de defesa por parte dos usuários.

Parasuraman (2000) divide os sentimentos em relação às tecnologias, destacados por Mick e Fournier (1998), em dois aspectos: os estimuladores e os inibidores da prontidão para usar a tecnologia. Esses sentimentos dos usuários podem aumentar (exemplo: percepção da facilidade de uso) ou diminuir (exemplo: percepção de risco) a probabilidade de uso de novas tecnologias. Em cursos EaD, esses sentimentos podem afetar o desempenho do aluno e a sua capacidade de acompanhar as tarefas propostas, geralmente publicadas em ambientes virtuais de aprendizagem que utilizam diferentes ferramentas de interação tais como blogs, chats, fóruns, web conferências entre outros.

A interação está relacionada à capacidade do discente de se envolver nas atividades propostas no curso, síncronas ou assíncronas, geralmente via ambiente virtual de aprendizagem. Alunos que possuem maior propensão à interação e participam ativamente de interações de aprendizagem, especialmente com os professores e com tarefas propostas, são mais propensos a persistirem (MORRIS et al., 2005). Em cursos $\mathrm{EaD}$, é a interação que propicia ao aluno o sentimento de pertencimento e acolhimento.

Finalmente, o último nível do Modelo 3M, o mais concreto, inclui os traços superficiais que representam comportamentos altamente singulares, resultantes dos efeitos dos traços elementares, compostos e 
situacionais, bem como da pressão do contexto específico do ambiente. Os traços superficiais mostram diretamente a resposta a estímulos específicos ou à frequência de realização de comportamentos em um dado contexto. Na pesquisa, o traço superficial de interesse é a persistência discente.

Em síntese, o Modelo 3M propõe a combinação de traços dos diferentes níveis da hierarquia para influenciar, direta e/ou indiretamente, os resultados de um comportamento - no caso, nos interessa o comportamento de persistência em cursos superiores em EaD.

\section{Metodologia de pesquisa}

Para atingir o objetivo da pesquisa (qual seja, comparar traços de personalidade associados à persistência discente), foi utilizada a técnica delineamentos quase-experimentais, atribuindo-se indivíduos aos diferentes grupos, conforme suas características demográficas (renda, gênero, curso) e características dos cursos que frequentam (grau e área do conhecimento). Dados dos quase-experimentos foram analisados pela técnica estatística ANOVA (análise de variância), conforme sugestão de Mowen, Harris e Bone (2004).

A primeira etapa da pesquisa consistiu em uma pesquisa bibliográfica para identificar escalas de operacionalização dos traços de personalidade. Incluiu a tradução de escalas e sua adaptação. A segunda etapa da pesquisa foi um estudo transversal, realizado por meio de levantamentos, baseado na aplicação de questionários, autopreenchidos em amostra representativa de discentes matriculados em cursos de nível superior de licenciatura e de especialização EaD da Universidade Federal do Espírito Santo (UFES), cujo banco de dados foi colocado à disposição dos pesquisadores.

O projeto de pesquisa foi submetido e aprovado pelo Comité de Ética em Pesquisa da UFES. Foram tomados cuidados associados, tais como: obtenção de consentimento livre e esclarecido dos respondentes e sujeitos experimentais, respeito por sua privacidade e eventual decisão de 
não completar sua participação e esclarecimento aos participantes sobre a pesquisa realizada.

Os traços de personalidade selecionados e as escalas de mensuração são apresentados no Anexo I. Para todos os itens foram utilizadas escalas do tipo Likert, variando de 1 ("raramente") a 7 ("sempre") para os traços elementares, e de 1 ("discordo totalmente") a 7 ("concordo totalmente") para os demais traços. Por se tratar de indicadores de traços de personalidade e de construtos psicológicos correlatos, questões correspondentes no instrumento de pesquisa utilizam escalas forçadas (sem a opção "não sei") e equilibradas (com ponto central de neutralidade).

Os construtos foram operacionalizados por meio de múltiplos indicadores, escolhidos com base em aplicações anteriores do Modelo 3M (LEMOS, 2017, SOPRANI, 2017, BENTO, 2016, MONTEIRO, 2006, 2010; MOWEN, 2000) e escalas disponíveis na literatura especializada sobre o tema (LIECHTENSTEIN; NETEMEYER; BURTON, 1990; SHIN, 2003; DUNCAN; MCKEACHIE, 2005; JAIN, 2013 e BLAZEVIC et al, 2014). Em função do grande número de construtos a serem mensurados simultaneamente, sua operacionalização deu-se a partir de escalas com poucos itens, conforme prática bem-sucedida nos estudos do Modelo 3M (MOWEN, 2000, MONTEIRO, 2010; MONTEIRO; VEIGA, 2006) em que as escalas utilizadas apresentam boas propriedades psicométricas.

\section{Apresentação e análise dos resultados}

Foram realizados dois estudos transversais de natureza quantitativa, sendo o primeiro com discentes de sete cursos de licenciatura e o segundo com discentes de quatro cursos de pós-graduação lato sensu (especialização), ambos na modalidade EaD oferecidos pela UFES no âmbito do Sistema Universidade Aberta do Brasil (UAB), da Coordenação de Aperfeiçoamento de Pessoal de Nível Superior (Capes). Os dois estudos foram levantamentos baseados na aplicação de questionários autopreenchidos pelos respondentes (em ambos, utilizou-se o mesmo formulário). A coleta de dados foi realizada pela internet, utilizando versão equivalente 
do questionário, postada nos ambientes virtuais de aprendizagem (AVA) dos cursos analisados, acessados diariamente pelos estudantes. A Tabela 1 faz o comparativo entre a população dos cursos analisados e amostra utilizada na pesquisa.

Tabela 1 - Comparativo entre Discentes dos Cursos e Discentes da Amostra

\begin{tabular}{|c|c|c|c|c|c|c|c|}
\hline Nível de Curso & Curso & $\begin{array}{l}\text { Matriculas } \\
\text { (M) }\end{array}$ & $\begin{array}{l}\text { Cursando } \\
\text { (C) }\end{array}$ & $\begin{array}{l}\text { \% Persistência } \\
\text { (C/M) }\end{array}$ & $\begin{array}{l}\text { Amostra } \\
\text { (A) }\end{array}$ & $\begin{array}{c}\% \\
(\mathrm{~A} / \mathrm{M})\end{array}$ & $\begin{array}{c}\% \\
(\mathrm{~A} / \mathrm{C})\end{array}$ \\
\hline \multirow{7}{*}{ Licenciatura } & História & 330 & 188 & $57,0 \%$ & 110 & 33,33 & 58,51 \\
\hline & Artes Visuais & 313 & 172 & $55,0 \%$ & 96 & 30,67 & 55,81 \\
\hline & Pedagogia & 438 & 357 & $81,5 \%$ & 89 & 20,32 & 24,93 \\
\hline & Filosofia & 317 & 179 & $56,5 \%$ & 81 & 25,55 & 45,25 \\
\hline & $\begin{array}{l}\text { Ciências } \\
\text { Biológicas }\end{array}$ & 192 & 115 & $59,9 \%$ & 73 & 38,02 & 63,48 \\
\hline & Física & 147 & 106 & $72,1 \%$ & 29 & 19,73 & 27,36 \\
\hline & Química & 74 & 27 & $36,5 \%$ & 12 & 16,22 & 44,44 \\
\hline \multicolumn{2}{|c|}{ Total das Licenciaturas } & 1.811 & 1.144 & $63,2 \%$ & 490 & 27,06 & 42,83 \\
\hline \multirow{4}{*}{ Especialização } & $\begin{array}{l}\text { Ensino de } \\
\text { Matemática }\end{array}$ & 240 & 238 & $99,2 \%$ & 21 & 8,75 & 8,82 \\
\hline & $\begin{array}{l}\text { Filosofia e } \\
\text { Psicanálise }\end{array}$ & 345 & 320 & $92,8 \%$ & 27 & 7,83 & 8,44 \\
\hline & $\begin{array}{l}\text { Gestão } \\
\text { Pública }\end{array}$ & 300 & 237 & $79,0 \%$ & 69 & 23,00 & 29,11 \\
\hline & Oratória & 500 & 352 & $70,4 \%$ & 165 & 33,00 & 46,88 \\
\hline \multicolumn{2}{|c|}{ Total das Especializações } & 1.385 & 1.147 & $82,8 \%$ & 282 & 20,36 & 24,59 \\
\hline
\end{tabular}

Nota: 1 ) siglas: $\mathrm{M}=$ Matriculados no início do curso; $\mathrm{C}=$ cursando no momento da pesquisa; $\mathrm{A}$ = participantes da amostra.

A amostra teve a participação de $42,83 \%$ dos estudantes de licenciatura e $24,59 \%$ dos estudantes de especialização vinculados à época da pesquisa ( $2^{\circ}$ semestre de 2017). Nos cursos de licenciatura em Ciências Biológicas, História e Artes Visuais, mais da metade dos discentes participou da pesquisa. Esses percentuais de participação são indicativos da significativa representatividade dos respondentes em relação aos cursos analisados. Ainda assim, generalizações dos resultados devem ser 
adotadas com cautela, sendo mais bem interpretadas como resultados preliminares, a serem utilizados como hipóteses de trabalho em estudos de maior amplitude e com amostras mais representativas.

As amostras do Estudo 1, com discente de cursos de licenciatura, e do Estudo 2, com alunos de cursos de especialização, tiveram, respectivamente, 490 e 282 questionários respondidos. Nos cursos de licenciatura, observa-se (Tabela 2) uma concentração de discentes na faixa etária entre 30 e 39 anos, confirmando uma característica dos cursos $\mathrm{EaD}$ de atraírem alunos não convencionais (com mais idade, que trabalham e que não cursaram o ensino superior logo após a conclusão do ensino médio). Observa-se uma predominância de estudantes do sexo feminino $(66,12 \%)$, casados ou com união estável $(61,02 \%)$ e com renda entre $R \$$ $1.021,00$ e $\mathrm{R} \$ 2.040,00$ (51,84\%), isto é, entre 1 e 2,5 salários mínimos, considerada baixa. A maioria dos discentes dos cursos de licenciatura atuam como professores da rede pública ou privada ${ }^{4}$.

Ainda na Tabela 2, observam-se as características dos 282 participantes dos cursos de especialização voltados para a formação continuada de professores de ensino fundamental e médio e formação de gestores públicos. É possível observar uma concentração de discentes na faixa etária entre 30 e 39 anos (41,49\%), do sexo feminino (68,79\%), casados ou com união estável $(66,31 \%)$ e com renda entre $\mathrm{R} \$ 2.041,00$ e $\mathrm{R} \$ 5.100,00$ (48,94\%), uma faixa de renda acima dos discentes da amostra do primeiro estudo. A maior participação foi de discentes do curso de Oratória (46,88\% dos alunos matriculados), que iniciou as aulas em maio de 2017, seguido pelo de Gestão Pública (29,11\%), que iniciou as aulas em abril de 2017. A baixa participação dos discentes dos cursos de Ensino de Matemática $(8,82 \%)$ e Filosofia e Psicanálise $(8,44 \%)$ pode ter ocorrido em função do início recente do curso (agosto e outubro de 2017, respectivamente). Todos os cursos de especialização EaD ofertados pela UFES via Sistema UAB têm 18 meses de duração, 12 dos quais para o curso das disciplinas e os 6 restantes para o Trabalho de Conclusão.

${ }^{4}$ Informação fornecida pela secretaria acadêmica dos cursos. 
Tabela 2 - Descrição da Amostra

(continua)

\begin{tabular}{|c|c|c|c|c|}
\hline \multirow[b]{2}{*}{ Idade } & \multicolumn{2}{|c|}{ Licenciatura } & \multicolumn{2}{|c|}{ Especialização } \\
\hline & $\mathbf{n}$ & $\%$ & $\mathbf{n}$ & $\%$ \\
\hline 18 a 29 anos & 141 & 28,78 & 38 & 13,48 \\
\hline 30 a 39 anos & 176 & 35,92 & 117 & 41,49 \\
\hline 40 a 49 anos & 118 & 24,08 & 79 & 28,01 \\
\hline 50 a 59 anos & 49 & 10 & 42 & 14,89 \\
\hline 60 anos ou mais & 6 & 1,22 & 6 & 2,13 \\
\hline Total & 490 & 100 & 282 & 100 \\
\hline Gênero & n & $\%$ & $\mathbf{n}$ & $\%$ \\
\hline Feminino & 324 & 66,12 & 194 & 68,79 \\
\hline Masculino & 166 & 33,88 & 88 & 31,21 \\
\hline Total & 490 & 100 & 282 & 100 \\
\hline Estado Civil & $\mathbf{n}$ & $\%$ & n & $\%$ \\
\hline Solteiro & 156 & 31,84 & 70 & 24,82 \\
\hline Casado/união estável & 299 & 61,02 & 187 & 66,31 \\
\hline Divorciado/Separado & 32 & 6,53 & 23 & 8,16 \\
\hline Viúvo & 3 & 0,61 & 2 & 0,71 \\
\hline Total & 490 & 100 & 282 & 100 \\
\hline Renda & $\mathbf{n}$ & $\%$ & $\mathbf{n}$ & $\%$ \\
\hline Entre $R \$ 1.021,00$ e $R \$ 2.040,00$ & 254 & 51,84 & 90 & 31,91 \\
\hline Entre $R \$ 2.041,00$ e $R \$ 5.100,00$ & 198 & 40,41 & 138 & 48,94 \\
\hline Entre $R \$ 5.101,00$ e $R \$ 10.200,00$ & 29 & 5,92 & 44 & 15 \\
\hline Acima de $\mathrm{R} \$ 10.201,00$ & 9 & 1,84 & 10 & 3,55 \\
\hline Total & 490 & 100 & 282 & 100 \\
\hline
\end{tabular}




\begin{tabular}{lcccc}
\hline Curso & $\mathbf{n}$ & $\%$ & $\mathbf{n}$ & $\%$ \\
\hline História & 110 & 22,45 & & \\
Artes Visuais & 96 & 19,59 & & \\
Pedagogia & 89 & 18,16 & & \\
Filosofia & 81 & 16,53 & & \\
Biologia & 73 & 14,9 & & \\
Física & 29 & 5,92 & & \\
Química & 12 & 2,45 & & 24,51 \\
Oratória & & & 69 & 9,57 \\
Gestão Pública & & & 21 & 7,45 \\
Filosofia e Psicanálise & & & $\mathbf{2 8 2}$ & $\mathbf{1 0 0}$ \\
Ensino de Matemática & & & & \\
\hline Total & 490 & 100 & & \\
\hline
\end{tabular}

Fonte: Elaboração própria (2017).

Todos os cursos de licenciatura iniciaram no segundo semestre letivo do ano de 2014. Os cursos EaD da UFES são ofertados em turmas únicas - uma nova turma somente inicia após a conclusão da turma anterior. Os cursos analisados na presente pesquisa, no momento da coleta dos dados, ofertavam o $7^{\circ}$ semestre letivo (aproximadamente metade do curso). Todos os cursos de especialização EaD desta pesquisa iniciaram em 2017. Foram ofertadas vagas nos polos de apoio presencial da região metropolitana de Vitória (Vila Velha e Vitória), onde há maior demanda por vagas. Há encontros presenciais quinzenais obrigatórios nos polos.

Após a descrição da amostra, na sequência, procedeu-se a análise fatorial confirmatória para testar a confiabilidade dos constructos que representam os traços de personalidade analisados. A Tabela 3 mostra que, para melhorar a confiabilidade dos constructos, foram excluídos 25 indicadores. Após ajustes, é possível verificar que todos os constructos atendem ao critério mínimo estabelecido por Hair et al. (2010), qual seja, o coeficiente Alpha de Crombach acima de 0,7. 
Tabela 3 - Resultado do Teste de Confiabilidade dos Constructos

\begin{tabular}{lccc}
\hline Traços & $\begin{array}{c}\text { Alfa de } \\
\text { Cronbach }\end{array}$ & $\begin{array}{c}\text { N. de indicadores } \\
\text { escala original }\end{array}$ & $\begin{array}{c}\text { N. de indicadores } \\
\text { após ajustes }\end{array}$ \\
\hline Abertura à Experiência (AE) & 0,754 & 4 & 3 \\
Instabilidade Emocional (IE) & 0,821 & 5 & 4 \\
Conscienciosidade (C) & 0,735 & 5 & 4 \\
Introversão (I) & 0,726 & 5 & 4 \\
Amabilidade (A) & 0,752 & 4 & 3 \\
Necessidade de Recursos Materiais (NRM) & 0,833 & 4 & 3 \\
Necessidade de Excitação (NEXC) & 0,802 & 5 & 5 \\
Necessidades de Recursos Corporais (NRC) & 0,825 & 4 & 3 \\
Autoeficácia (AF) & 0,816 & 4 & 2 \\
Necessidade de Aprendizado (NA) & 0,729 & 4 & 3 \\
Orientação para Tarefas (OT) & 0,743 & 4 & 2 \\
Satisfação (S) & 0,877 & 8 & 5 \\
Interação (IT) & 0,879 & 8 & 5 \\
Consciência de Valor (CV) & 0,856 & 7 & 5 \\
Aceitação Tecnologia (AT) & 0,701 & 10 & 5 \\
Persistência Discente (PD) & 0,827 & 6 & 4 \\
\hline Fonte: Elaboração própria (2017). & & & \\
& & &
\end{tabular}

Buscou-se, na sequência, identificar a heterogeneidade das amostras, analisando as diferenças de médias de grupos formados a partir de características demográficas dos participantes. Analisou-se, com procedimento ANOVA do SPSS ${ }^{\circledR}$, as diferenças de médias de cada um dos traços elementares, compostos, situacionais e superficial do modelo proposto.

A primeira análise comparou a diferença de médias para os grupos Gênero (feminino ou masculino), Estado Civil (solteiro, casado ou separado), Renda (menos de $\mathrm{R} \$ 2$ mil, entre $\mathrm{R} \$ 2$ mil e $\mathrm{R} \$ 5$ mil e acima de R $\$ 5$ mil), Grau do Curso (licenciatura ou especialização), Área de Conhecimento do Curso (Humanidades e Ciências Sociais ou Ciências Exatas e Biológicas). Os resultados estão apresentados na Tabela 4. Foi considerado intervalo de confiança de $95 \%$.

A primeira análise comparou os traços de personalidade de grupos com diferenças pessoais (gênero, estado civil e renda). Os traços abertura à experiência, instabilidade emocional e interação não apresentaram 
diferença de médias estatisticamente significativas para qualquer dos grupos analisados, indicando não haver diferenças internas desses traços.

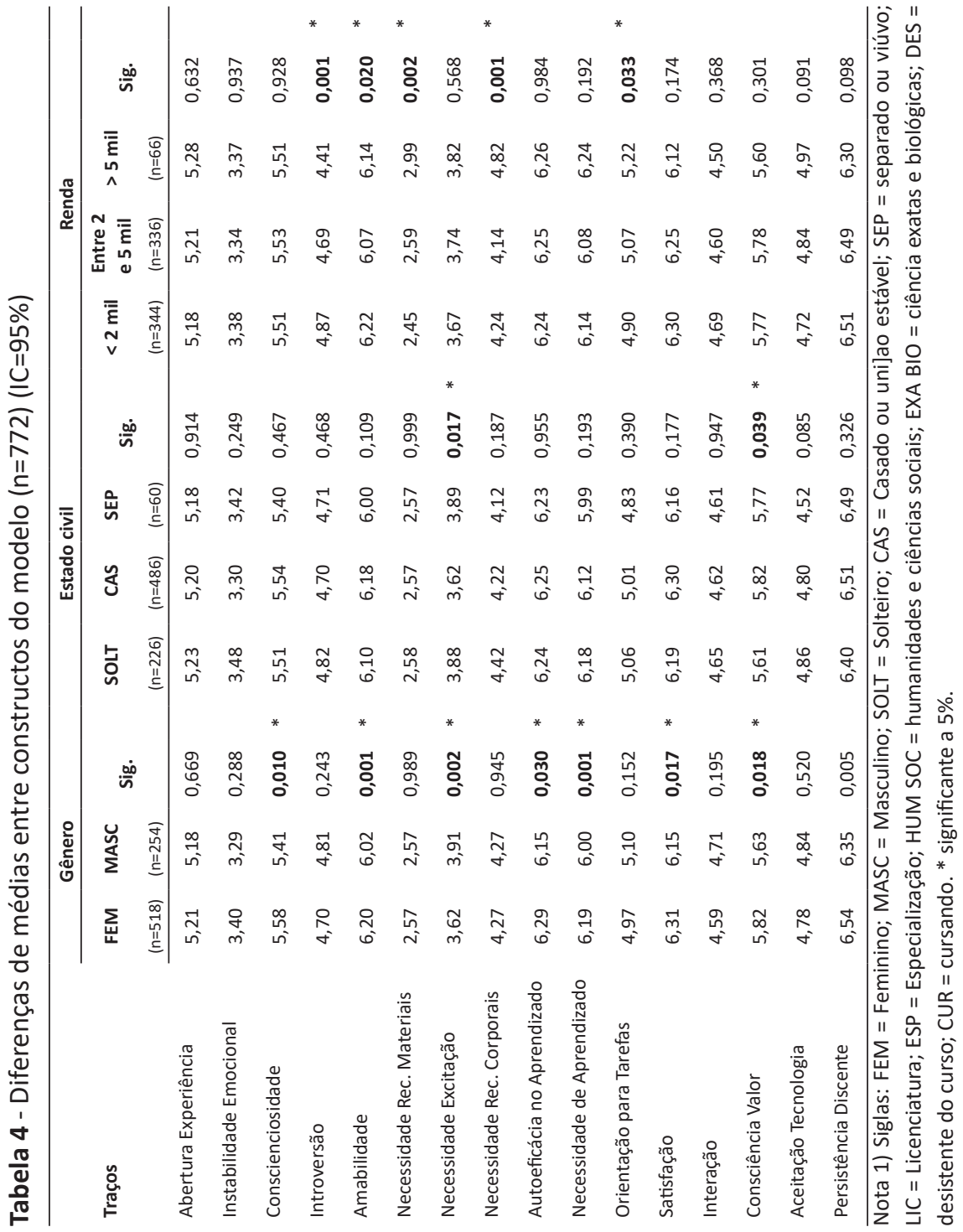


Comparando os grupos por gênero, estudantes do gênero feminino são significativamente mais amáveis, mais conscienciosas, têm menos necessidade de excitação, mais necessidade de aprendizado, são mais autoeficazes no aprendizado, possuem mais consciência de valor, estão mais satisfeitas com os cursos e são mais persistentes que os discentes do gênero masculino. Esses resultados estão parcialmente alinhados a resultados de pesquisa realizada por South, Jarnecke e Vize (2018) que apontou diferenças em três dos cinco traços de personalidade (instabilidade emocional, amabilidade, consciência), mas não encontrou diferenças estatisticamente significantes nos traços introversão e abertura à experiência. Também se alinham parcialmente aos achados de Kajonius e Johnson (2018) que identificaram que as mulheres pontuam mais alto que os homens em amabilidade e instabilidade emocional, enquanto os homens pontuam um pouco mais do que as mulheres em traços associados ao intelecto como a assertividade.

Comparando os grupos por estado civil, os traços necessidade de excitação e consciência de valor apresentaram diferença estatisticamente significante entre os três grupos - casados possuem maior consciência de valor e menos necessidade de excitação do que solteiros e separados/ divorciados/viúvos.

Estudo de Lavner et al. (2018), que investigou traços de personalidade e estado civil usando dados coletados nos primeiros 18 meses de casamento, indicou mudanças significativas na personalidade ao longo do tempo do matrimônio, incluindo declínios em amabilidade para maridos e para esposas, declínios na extroversão para maridos, declínios na abertura e instabilidade emocional para as esposas e aumento da conscienciosidade para os maridos. Os estudos não podem ser comparados, embora os resultados do estudo de Lavner et al. (2018) reforcem a associação dos traços de personalidade com o estado civil.

Na comparação dos grupos por faixa de renda mensal, as diferenças significativas identificadas foram nos traços amabilidade, necessidade de recursos corporais e introversão. Grupos que se situam em faixa de renda intermediária são menos amáveis e possuem menor necessidade 
de recursos corporais do que os grupos que se situam nos extremos das faixas de renda - menos de $\mathrm{R} \$ 2$ mil ou mais de $\mathrm{R} \$ 5$ mil. O traço introversão diminui com o aumento da renda. Indivíduos que se encontram na faixa de renda mais baixa apresentam menor necessidade de recursos materiais e são menos orientados a tarefa do que indivíduos classificados nos grupos de maior renda. Quanto maior a renda, maior a necessidade de recursos materiais, maior a orientação para tarefas e menor a introversão.

A Tabela 5 mostra que a maioria dos estudantes dos cursos de licenciatura (51,8\%) estão classificados na faixa de mais baixa renda mensal (< R \$ 2 mil) e apenas uma minoria (19,1\%) está na faixa de mais alta renda ( $>$ R\% 5 mil). Já nas especializações, a maior parte $(48,9 \%)$ dos estudantes está na faixa intermediária de renda, embora uma parcela significativa (31,9\%) esteja na faixa de mais baixa renda. Esses dados alertam para o perfil desses discentes que, por terem baixa renda, podem apresentar menor orientação para as tarefas e maior introversão, dois traços importantes para o comportamento de persistência em cursos EaD. Esses resultados corroboram com Tinto (2012), que identificou que alunos de renda elevada têm três vezes mais chances de concluir o ensino superior em comparação àqueles de baixa renda.

Tabela 5 - Renda x Curso - Tabulação Cruzada

\begin{tabular}{lcccc}
\hline Renda Mensal/ Curso & \multicolumn{2}{c}{ Licenciaturas } & \multicolumn{2}{c}{ Especializações } \\
\hline < R\$ 2 mil & 254 & $51,8 \%$ & 90 & $31,9 \%$ \\
Entre R\$ 2 e R\$ 5 mil & 198 & $40,4 \%$ & 138 & $48,9 \%$ \\
$>$ R\$ 5 mil & 38 & $7,8 \%$ & 54 & $19,1 \%$ \\
\hline Total & 490 & $100,0 \%$ & 282 & $100,0 \%$ \\
\hline
\end{tabular}

Fonte: Elaboração própria (2017).

A segunda análise comparou a diferença de médias para os grupos: Grau do Curso (licenciatura ou especialização) e Área de Conhecimento do Curso (Humanidades e Ciências Sociais ou Ciências Exatas e Biológicas). Os resultados estão apresentados na Tabela 6. Os 
traços instabilidade emocional, introversão e interação, necessidade de excitação, autoeficácia no aprendizado, necessidade de aprendizado, orientação para tarefas e satisfação não apresentaram diferença de média estatisticamente significante para quaisquer dos grupos analisados.

Na comparação entre os cursos, discentes de especialização são mais abertos a experiências, mais conscienciosos, possuem mais necessidade de recursos materiais e corporais, são mais interativos, aceitam mais a tecnologia e possuem maior consciência de valor. Não foram encontrados estudos comparando traços de personalidade e escolaridade. Hopwood et al. (2011) analisaram a mudança de traços de personalidade com a idade. Durante a transição para a idade adulta, os indivíduos normalmente se estabelecem em papéis de maior responsabilidade nos relacionamentos e no trabalho. Essa transição envolve mudanças significativas nos traços de personalidade devido às transações com o ambiente social. A tendência, portanto, é de aumento da maturidade da personalidade. Sendo assim, os resultados da presente pesquisa corroboram com os achados de Hopwood et al. (2011).

Tabela 6 - Diferenças de médias entre constructos do modelo ( $n=772)$ (IC=95\%)

(continua)

\begin{tabular}{|c|c|c|c|c|c|c|c|c|}
\hline \multirow[b]{2}{*}{ Traços } & \multicolumn{3}{|c|}{ Grau do Curso } & & \multicolumn{4}{|c|}{ Área do Curso } \\
\hline & $\begin{array}{c}\text { LIC } \\
(n=490)\end{array}$ & $\begin{array}{c}\text { ESP } \\
(n=282)\end{array}$ & Sig. & & $\begin{array}{l}\text { HUM SOC } \\
(n=637)\end{array}$ & $\begin{array}{l}\text { EXA BIO } \\
(n=135)\end{array}$ & Sig. & \\
\hline Abertura Experiência & 5,15 & 5,30 & 0,036 & $*$ & 5,25 & 4,97 & 0,002 & $*$ \\
\hline Instabilidade Emocional & 3,42 & 3,27 & 0,136 & & 3,35 & 3,43 & 0,516 & \\
\hline Conscienciosidade & 5,47 & 5,62 & 0,018 & $*$ & 5,56 & 5,36 & 0,015 & $*$ \\
\hline Introversão & 4,73 & 4,75 & 0,840 & & 4,74 & 4,72 & 0,815 & \\
\hline Amabilidade & 6,16 & 6,11 & 0,316 & & 6,17 & 6,02 & 0,031 & $*$ \\
\hline $\begin{array}{l}\text { Necessidade Recursos } \\
\text { Materiais }\end{array}$ & 2,50 & 2,70 & 0,043 & $*$ & 2,62 & 2,35 & 0,026 & $*$ \\
\hline $\begin{array}{l}\text { Necessidade de } \\
\text { Excitação }\end{array}$ & 3,68 & 3,78 & 0,309 & & 3,68 & 3,87 & 0,099 & \\
\hline $\begin{array}{l}\text { Necessidade de Recursos } \\
\text { Corporais }\end{array}$ & 4,16 & 4,46 & 0,008 & $*$ & 4,30 & 4,12 & 0,195 & \\
\hline $\begin{array}{l}\text { Autoeficácia no } \\
\text { Aprendizado }\end{array}$ & 6,23 & 6,27 & 0,530 & & 6,26 & 6,17 & 0,229 & \\
\hline
\end{tabular}


(conclusão)

\begin{tabular}{lccccccc}
\hline & \multicolumn{3}{c}{ Grau do Curso } & \multicolumn{3}{c}{ Área do Curso } \\
\cline { 2 - 8 } Traços & $\begin{array}{c}\text { LIC } \\
(\mathbf{n = 4 9 0 )}\end{array}$ & $\begin{array}{c}\text { ESP } \\
(\mathbf{n = 2 8 2})\end{array}$ & Sig. & $\begin{array}{c}\text { HUM SOC } \\
(\mathbf{n = 6 3 7 )}\end{array}$ & $\begin{array}{c}\text { EXA BIO } \\
(\mathbf{n = 1 3 5 )}\end{array}$ & Sig. \\
\hline $\begin{array}{l}\text { Necessidade de } \\
\text { Aprendizado }\end{array}$ & 6,11 & 6,15 & 0,426 & 6,15 & 6,02 & 0,082 \\
Orientação para Tarefas & 4,98 & 5,07 & 0,274 & 5,03 & 4,91 & 0,282 \\
Satisfação & 6,27 & 6,23 & 0,497 & & 6,28 & 6,14 & 0,081 \\
Interação & 4,57 & 4,73 & 0,069 & $*$ & 4,64 & 4,58 & 0,612 \\
Consciência Valor & 5,70 & 5,86 & 0,047 & $*$ & 5,80 & 5,57 & 0,022 \\
Aceitação Tecnologia & 4,74 & 4,90 & 0,051 & $*$ & 4,80 & 4,79 & 0,926 \\
Persistência Discente & 6,48 & 6,47 & 0,925 & & 6,49 & 6,42 & 0,405 \\
\hline
\end{tabular}

Nota 1) Siglas: FEM = Feminino; MASC = Masculino; SOLT = Solteiro; CAS = Casado ou uni]ao estável; SEP = separado ou viúvo; LIC = Licenciatura; ESP = Especialização; HUM SOC = humanidades e ciências sociais; EXA BIO = ciência exatas e biológicas; DES = desistente do curso; CUR = cursando. * significante a 5\%.

Quando comparados os traços de personalidade pelas áreas de conhecimentos dos cursos em que estão matriculados, discentes de cursos das áreas de Humanidades e Ciências Sociais são mais amáveis, mais abertos a experiências, mais conscienciosos e possuem maior necessidade de recursos materiais e maior consciência de valor quando comparados aos discentes das áreas de Ciências Exatas e Biológicas. Carneiro (2017), em estudo que examinou os traços de personalidade como antecedentes do comportamento de persistência discente em cursos superiores na modalidade a distância, identificou que a amabilidade, a autoeficácia, a necessidade de aprendizado e a satisfação com o curso são os traços que mais bem explicam a persistência discente nessa modalidade de ensino. Sendo assim, os resultados apresentados na Tabela 6 indicam haver maior propensão à persistência em cursos EaD em Humanidades e Ciências Sociais, dado que os traços associados à persistência são maiores nos discentes de cursos destas áreas, para a amostra analisada.

\section{Considerações finais}

Apesar de evidências gerais satisfatórias de confiabilidade e validade dos construtos que representam os traços de personalidade 
analisados, enfatizamos a necessidade de melhorias nos indicadores dos traços autoeficácia, orientação para tarefas e aceitação de tecnologia, para aumentar a consistência interna e validade convergente para estudos relacionados a comportamento de discentes.

Devido ao perfil das amostras dos dois estudos (estudantes de cursos superiores de formação de professores que atuam como professores do ensino fundamental e médio e de profissionais do setor público, matriculados em universidade pública e gratuita), os resultados não podem ser generalizados para outras subpopulações mais representativas da população brasileira (cursos ofertados por instituições privadas, de bacharelado, ou cursos de extensão ou de curtíssima duração). Esse é um perfil importante a ser investigado, com amostras representativas, em estudos futuros.

A presente pesquisa, ao investigar dois diferentes tipos de curso, um mais longo e de formação inicial (licenciaturas) e outro mais curto e de formação mais avançada (especialização), permitiu analisar o grau em que diferentes traços da personalidade são aplicáveis para diferentes situações.

Como aplicação prática dos resultados da pesquisa, estratégias para promover programas de apoio didático e psicológico poderiam enfatizar ações para discentes com baixa amabilidade, baixa autoeficácia, baixa necessidade de aprendizado e insatisfação com o curso. No caso de cursos das áreas de Ciências Exatas e Biológicas, seria recomendável enfocar a baixa interação com professores e colegas de turma. Se os resultados obtidos puderem ser generalizados, incorporar à rotina de orientação discente, por parte de professores, tutores e coordenadores de curso, um programa de acompanhamento particularizado àqueles com baixa pontuação nesses traços de personalidade.

Para cursos de especialização, em que o processo seletivo não se dá por exame de conhecimentos (do tipo exame vestibular), poderia ser incluída uma avaliação prévia do perfil de personalidade dos discentes, para que se pudesse desenvolver uma análise da propensão do discente a permanecer no curso. Essa estratégia seria especialmente relevante em 
cursos públicos e gratuitos, evitando-se, assim, uma destinação incorreta ou menos apropriada de recursos públicos. Altos níveis de evasão em cursos superiores públicos representam uma alocação ineficiente de recursos escassos destinados à educação; além disso, se contrastam com a função da universidade como instituição social e com a sua missão de formar e de buscar meios de permanência dos estudantes.

A universidade brasileira, em particular a universidade pública, tem sido enfaticamente questionada pela retenção discente. A expansão do ensino superior, em contraste com a má qualidade da formação na educação básica e com o despreparo das universidades, em face disso, impõe desafios cada vez mais constantes à criação de estratégias de combate à evasão e de apoio à permanência dos estudantes.

A esse propósito, estudos como o que aqui apresentamos revestem-se de importância, no mínimo, para parametrizar um debate, ainda difuso e não concertado, mas necessário e estratégico ao (re)desenho institucional da formação superior no País.

\section{Referências}

ALENCAR, L. M. B. A evasão discente no contexto da reestruturação universitária: o caso dos cursos de administração e ciências contábeis da Universidade Federal do Espírito Santo. Dissertação (Mestrado em Gestão Pública) — Universidade Federal do Espírito Santo, Vitória/ES, 2014.

BEAN, J. P. Dropouts and turnover: the synthesis and test of a causal model of student attrition. Research in Higher Education, v. 12, n. 2, p. 155-187, 1980.

BEAN, J. P.; METZNER, B. S. A conceptual model of nontraditional undergraduate student attrition. Review of Educational Research, v. 55, p. 485-540, 1985.

BLAZEVIC, V.; WIERTZ, C.; COTTE, J.; RUYTER, K.; ISOBEL, K. D. Gosip in cyberspace: conceptualization and scale development for general online social interaction propensity. Journal of Interactive Marketing, v. 28, n. 2, p. 87-100, 2014. 
BENTO, F. C. Papel moderador do tipo de produto na relação entre traços de personalidade e comportamento de compra online: uma aplicação do modelo hierárquico de Mowen. Dissertação (Mestrado em Administração) Universidade Federal do Espírito Santo, Vitória/ES, 2016.

BUSS, A. Personality: evolutionary heritage and human distinctiveness. Hillsdale: Lawrence Erlbaum Associates, 1988.

CARNEIRO, T. C. J. Modelo hierárquico dos antecedentes do comportamento de persistência discente em cursos superiores na modalidade a distância. Relatório Final de Pesquisa. Vitória, 2017. Projeto submetido à Chamada Universal - MCT/ CNPq No. 14/2014.

CARVER, C. S.; SCHEIRER, M. F. Origins and functions of positive and negative affect: a control-process view. Psychological Review, v. 97, n. 1, p. 19-35, 1990.

CHUNG, H. J. The study on learning persistence and non-persistence factors of distance learners. The Korean Journal for Human Resource Development, v. 2, n. 1, p. 173-188, 2000.

DUNCAN, T. G.; McKEACHIE, W. J. The making of the motivated strategies for learning questionnaire. Educational Psychologist, v. 40, p. 117-128, 2005.

FINNEGAN, C., MORRIS, L. V., LEE, K. Differences by course discipline on student behavior, persistence, and achievement in online courses of undergraduate general education. Journal of College Student Retention: Research, Theory and Practice, v. 10, n. 1, p. 39-54, 2008.

FISHBEIN, M.; AJZEN, I. Belief, attitude, intention, and behavior: an introduction to theory and research. Reading: Addison-Wesley, 1975.

HAIR JUNIOR., J. F.; ANDERSON, R. E.; TATHAM, R. L.; BLACK, W. C. Multivariate data analysis, 7. ed. Upper Saddle River (NJ): Prentice-Hall, 2010.

HENRY, T. C.; SMITH, G. P. Planning student success and persistence: implementing a state system strategy. Community College Review, v. 22, n. 2, p. 26-36, 1993. 
HESS, F. M.; SCHNEIDER, M.; CAREY, K.; KELLY, A. P. Diplomas and dropouts: Which colleges actually graduate their students (and which don't). Washington: American Enterprise Institute, 2009. Disponível em: <http://www.aei.org/ files/2009/06/03/Diplomasand Dropoutsfinal.pdf>. Acesso em: 10 mai. 2014.

HOLDER, B. An investigation of hope, academics, environment, and motivation as predictors of persistence in higher education online programs. Internet and Higher Education, v. 10, n 4, p. 245-260, 2007.

HOPWOOD, C. J.; DONNELLAN, M. B.; BLONIGEN, D. M.; KRUEGER, R. F.; MCGUE, M.; IACONO, W. G.; BURT S. A. Genetic and environmental influences on personality trait stability and growth during the transition to adulthood: a three-wave longitudinal study. Journal of Personality and Social Psychology, v. 100, n. 3, p. 545-556, 2011.

JAIN, P. Technology readiness index measuring railway employee's readiness to embrace online technology. Journal of Marketing \& Communication, v. 8, n. 4, p. 52-56, 2013.

JOO, Y. J., JOUNG, S., SIM, W. J. Structural relationships among internal locus of control, institutional support, flow, and learner persistence in cyber universities. Computers in Human Behavior, v. 27, n. 2, p. 714-722, 2011.

JOO, Y. J., LIM, K. Y., KIM, E. K. Online university students' satisfaction and persistence: Examining perceived level of presence, usefulness and ease of use as predictors in a structural model. Computers and Education, v. 57, p. 1654-1664, 2011.

KAJONIUS, P. J.; JOHNSON, J. Sex differences in 30 facets of the five-factor model of personality in the large public $(\mathrm{N}=320,128)$. Personality and Individual Differences, v. 129, p. 126-130, 2018.

LAVNER, J. A.; WEISS, B.; MILLER, J. D.; KARNEY, B. R. Personality change among newlyweds: patterns, predictors, and associations with marital satisfaction over time. Developmental Psychology, v. 54, n. 6, p. 1172-1185, 2018. 
LEE, S. W. Comparing learners with institutions' factors that influence on persistence in university lifelong education. Journal of Lifelong Education, v. 9, n. 1, p. 117-148, 2003.

LEMOS, L. T. Relação entre traços de personalidade e comportamento de persistência discente em cursos de graduação na modalidade a distância. Dissertação (Mestrado em Gestão Pública) - Universidade Federal do Espírito Santo, Vitória/ES, 2017.

LEVY, Y. Comparing dropouts and persistence in e-learning courses. Computers and Education, v. 48, n. 2, p. 185-204, 2007.

McCRAE, R. R; COSTA, P. T. Personality trait structure as a human universal. American Psychologist, v. 52, n. 5, p. 509-516, 1997.

METZNER, B. S; BEAN, J. The estimation of a conceptual model of nontraditional undergraduate student attrition. Research in Higher Education, v. 27, p. 15-38, 1987.

MONTEIRO, P. R. Estratégias de segmentação de consumidores ecologicamente conscientes: uma abordagem fundamentada no Modelo 3M de Motivação e Personalidade. Tese (Doutorado em Administração) - Universidade Federal de Minas Gerais, 2010.

MONTEIRO, P. R.; VEIGA, R. T. Personalidade e consumo: uma avaliação empírica do modelo metateórico de motivação e personalidade. In: ENCONTRO NACIONAL DA ANPAD. 30 , Salvador, 2006. Anais... Rio de Janeiro: ANPAD, 2006.

MORRIS, L. V., FINNEGAN, C. L. Best practices in predicting and encouraging student persistence and achievement online. Journal of College Student Retention: Research, Theory and Practice, v. 10, n. 1, p. 55-64, 2008.

MORRIS, L. V.; WU, S. S.; FINNEGAN, C. L. Predicting retention in online general education courses. American Journal of Distance Education, v. 19, n.1, p.23-36, 2005. 
MORTENSEN, T. G. Measurements of persistence. In: SEIDMAN, A. (Org.). College student retention. The ACE Series of Higher Education, 2. ed., Rowman \& Littlefield, 2012.

MOWEN, J. C. The 3M Model of Motivation and Personality: theory and empirical applications to consumer behavior. Boston: Kluer Academic Publishers, 2000.

MOWEN, J. C.; VOSS, K. E. On building better construct measures: implications of a general hierarchical model. Psychology \& Marketing, v.25, n.6, p.485-505, 2008.

MÜLLER, T. Persistence of women in online degree-completion programs. International Review of Research in Open and Distance Learning, v. 9, n. 2, p. 1-18, 2008.

NISTOR, N.; NEUBAUER, K. From participation to dropout: quantitative participation patterns in online university courses. Computers and Education, v. 55, n. 2, p. 663-672, 2010.

PARASURAMAN, A. Technological Readiness Index: a multiple-item scale to measure readiness to embrace new technologies. Journal of Service Research, v. 2, n. 4, p. 307-320, 2000.

PARK, C. L.; PERRY, B.; EDWARDS, M. Minimising attrition: strategies for assisting students who are at risk of withdrawal. Innovations in Education and Teaching International, v. 48, n. 1, p. 37-47, 2011.

PAUNONEN, S. V. Hierarchical organization of personality and prediction of behavior. Journal of Personality and Social Psychology, v. 74, p. 538-556, 1998.

PEREIRA, A. S. Retenção discente nos cursos presencial da UFES. Dissertação (Mestrado em Gestão Pública) - Universidade Federal do Espírito Santo, Vitória, 2013.

PEREIRA, A. S.; CARNEIRO, T. C. J. ; BRASIL, G. H.; CORASSA, M. A. C. Principais características dos alunos retidos dos cursos de graduação presencial da Universidade Federal do Espírito Santo. Revista Gestão Universitária da América Latina, v. 9, n. 2, p. 238-259, 2016. 
PETRIS, W. G. C. Fatores que afetam a persistência dos discentes em cursos superiores na modalidade a distância. Dissertação (Mestrado em Gestão Pública) - Universidade Federal do Espírito Santo, Vitória /ES, 2014.

PITTENGER, A.; DOERING, A. Influence of motivational design on completion rates in online self- study pharmacy-content courses. Distance Education, v. 31, n. 3, p. 275-293, 2010.

ROVAI, A. P. In search of higher persistence rates in distance education online programs. The Internet and Higher Education, v. 6, p. 1-16, 2003.

SALES JUNIOR, J. S. Uma análise estatística dos fatores de evasão e permanência de estudantes presencial da UFES. Dissertação (Mestrado em Gestão Pública) Universidade Federal do Espírito Santo, Vitória, 2013.

SHIN, N. Transactional presence as a critical predictor of success in distance learning. Distance Education, v. 24, n. 1, p. 69-86, 2003.

SILVA FILHO, R. L. L. A evasão no ensino superior brasileiro: novos dados. In: Instituto Lobo, out, 2017. Disponível em: <http://www.institutolobo.org.br/ imagens/pdf/artigos/art_088.pdf >. Acesso em: 23 out. 2018.

SOPRANI, A. D. R. O uso de traços comportamentais na explicação da persistência discente em cursos de licenciatura ofertados na modalidade a distância pela UFES. Dissertação (Mestrado em Gestão Pública) - Universidade Federal do Espírito Santo, Vitória /ES, 2017.

SOUTH, S.C., JARNECKE, A. M., VIZE, C.E. Sex differences in the Big Five model personality traits: A behavior genetics exploration. Journal of Research in Personality, v. 74, p. 158-165, 2018.

TINTO, V. Leaving college: rethinking the causes and cures of student attrition. Chicago: University of Chicago Press, 1993.

TINTO, V. Completing college: rethinking institutional action. Chicago: University of Chicago Press, 2012. 
TINTO, V. Dropout from higher education: a theoretical synthesis of recent research. Review of Educational Research, v. 45, n. 1, p. 89-125, 1975.

TINTO, V. Leaving college: rethinking the causes and cures of student attrition. Chicago: University of Chicago Press, 1987.

ZUCKERMAN, M. Attribution of success and failure revisited, or: The motivational bias is alive and well in attribution theory. Journal of personality, v. 47, n. 2, p. 245-287, 1979.

Recebido: 11/11/2018

Received: 11/11/2018

Aprovado: 04/02/2019

Approved: 02/04/2019 


\section{ANEXO I \\ Escalas utilizadas na pesquisa}

Quadro 3 - Constructos e Indicadores utilizados

\begin{tabular}{|c|c|}
\hline Constructo & Indicadores \\
\hline \multirow{4}{*}{$\begin{array}{l}\text { Abertura à } \\
\text { Experiência }\end{array}$} & Criativo(a) \\
\hline & Sou mais original que os outros \\
\hline & Encontro soluções inovadoras para os problemas que enfrento \\
\hline & Frequentemente me sinto altamente criativo(a) \\
\hline \multirow{5}{*}{$\begin{array}{l}\text { Instabilidade } \\
\text { Emocional }\end{array}$} & Temperamental \\
\hline & Fico irritado(a) com facilidade \\
\hline & Meu humor muda de repente \\
\hline & Sou mais mal humorado(a) que os outros \\
\hline & Sou mais impaciente que os outros \\
\hline \multirow{5}{*}{ Conscienciosidade } & Metódico(a) - faço sempre as mesmas coisas \\
\hline & Minucioso(a) - preocupo-me com detalhes \\
\hline & Organizado(a) \\
\hline & Eficiente - consigo realizar minhas tarefas \\
\hline & Sistemático(a) - tenho regras para realizar minhas tarefas \\
\hline \multirow{5}{*}{ Introversão } & Introvertido(a) \\
\hline & Tímido(a) \\
\hline & Reservado(a) \\
\hline & Sou discreto(a) quando estou com outras pessoas \\
\hline & Prefiro estar sozinho(a) a estar num grande grupo de pessoas desconhecidas \\
\hline \multirow{4}{*}{ Amabilidade } & Compreensivo(a) \\
\hline & Sou atencioso(a) com os outros \\
\hline & Sou gentil com os outros \\
\hline & Sinto-me bem ao ajudar os outros \\
\hline \multirow{4}{*}{$\begin{array}{l}\text { Necessidade } \\
\text { de Recursos } \\
\text { Materiais }\end{array}$} & Gosto de comprar coisas caras \\
\hline & Gosto de ter artigos de luxo \\
\hline & Adquirir coisas de valor é importante para mim \\
\hline & Aprecio ter objetos de luxo mais do que outras pessoas \\
\hline
\end{tabular}




\begin{tabular}{|c|c|}
\hline Constructo & Indicadores \\
\hline \multirow{5}{*}{$\begin{array}{l}\text { Necessidade de } \\
\text { Excitação }\end{array}$} & Procuro atividades que me ofereçam adrenalina e aventura \\
\hline & Gosto de me arriscar mais do que os outros \\
\hline & Sinto-me atraído por experiências que têm um elemento de perigo \\
\hline & Prefiro coisas novas e diferentes a conhecidas e seguras \\
\hline & Gosto de assumir riscos nas atividades que realizo \\
\hline \multirow{4}{*}{$\begin{array}{l}\text { Necessidades } \\
\text { Físicas/de } \\
\text { Recursos } \\
\text { Corporais }\end{array}$} & Presto atenção no meu corpo e em minha aparência \\
\hline & Dedico um tempo cada dia para cuidar da minha forma física \\
\hline & Acho importante manter meu corpo em forma \\
\hline & Esforço-me para manter meu corpo saudável \\
\hline \multirow{4}{*}{$\begin{array}{l}\text { Necessidade de } \\
\text { Aprendizado }\end{array}$} & Gosto de aprender coisas novas mais do que as outras pessoas \\
\hline & Conhecimento é meu recurso mais importante \\
\hline & Divirto-me ao obter novos conhecimentos \\
\hline & Adquirir novos conhecimentos é essencial para a minha vida \\
\hline \multirow{4}{*}{$\begin{array}{l}\text { Orientação para } \\
\text { Tarefas }\end{array}$} & Sou orientado para objetivos de longo prazo \\
\hline & Estabeleço um prazo para terminar as tarefas que estou fazendo \\
\hline & Estabeleço objetivos de longo prazo \\
\hline & Os meus esforços determinam meu sucesso \\
\hline \multirow{4}{*}{ Autoeficácia } & Certamente, posso compreender os textos mais difíceis do meu curso EaD \\
\hline & $\begin{array}{l}\text { Tenho dúvidas se posso fazer um excelente trabalho nas tarefas e testes } \\
\text { aplicados no meu curso EaD }\end{array}$ \\
\hline & Sei que posso fazer bem meu curso EaD \\
\hline & $\begin{array}{l}\text { Considerando a dificuldade do curso, os professores e minhas habilidades, sei } \\
\text { que possuo capacidade de completar o meu curso EaD }\end{array}$ \\
\hline \multirow{8}{*}{ Satisfação } & Estudar é uma experiência valiosa para mim \\
\hline & Consegui aprender muito no curso EaD \\
\hline & Não me arrependo de ter me matriculado no curso EaD \\
\hline & $\begin{array}{l}\text { Sinto que estou crescendo de forma contínua, devido às atividades das quais } \\
\text { participei no meu curso EaD }\end{array}$ \\
\hline & Sinto que estou realizando algo importante enquanto faço o meu curso EaD \\
\hline & Gosto/Gostava de estudar no meu curso EaD \\
\hline & Vale/Valia a pena estudar no meu curso EaD \\
\hline & Incentivo outras pessoas a estudar em cursos EaD \\
\hline
\end{tabular}




\begin{tabular}{|c|c|}
\hline Constructo & Indicadores \\
\hline \multirow{8}{*}{ Interação } & Em geral, procuro manter contato on-line com as pessoas \\
\hline & Em geral, respondo questões de outras pessoas em fóruns de discussão on-line \\
\hline & Em geral, gosto de iniciar conversas on-line \\
\hline & Em geral, gosto de me envolver em discussões on-line \\
\hline & Acho agradável a ideia de pertencer a um grupo de discussão on-line \\
\hline & Gosto de participar ativamente de discussões on-line \\
\hline & Gosto de interagir de forma on-line com outras pessoas \\
\hline & Em geral, gosto de trocar ideias on-line com outras pessoas \\
\hline \multirow{7}{*}{$\begin{array}{l}\text { Consciência de } \\
\text { Valor }\end{array}$} & Ao comprar um produto, tento maximizar a qualidade obtida pelo valor gasto \\
\hline & $\begin{array}{l}\text { Preocupo-me muito com preços baixos, mas sou igualmente preocupado } \\
\text { com a qualidade dos produtos }\end{array}$ \\
\hline & $\begin{array}{l}\text { Nas compras de supermercado, comparo preços de diferentes marcas para } \\
\text { ter certeza de que vou obter o melhor valor }\end{array}$ \\
\hline & $\begin{array}{l}\text { Quando faço compras, gosto de ter certeza que estou obtendo o melhor } \\
\text { custo-benefício }\end{array}$ \\
\hline & $\begin{array}{l}\text { Procuro produtos mais baratos para comprar, mas eles devem atender certas } \\
\text { exigências de qualidade para que eu os adquira }\end{array}$ \\
\hline & $\begin{array}{l}\text { Quando faço compras, normalmente comparo o preço por unidade, volume } \\
\text { ou peso das marcas que compro }\end{array}$ \\
\hline & $\begin{array}{l}\text { Sempre verifico preços no supermercado para ter certeza de estar obtendo o } \\
\text { melhor valor pelo produto }\end{array}$ \\
\hline \multirow{10}{*}{$\begin{array}{l}\text { Aceitação da } \\
\text { Tecnologia }\end{array}$} & O uso da internet na prestação de serviços é muito empolgante \\
\hline & O uso de tecnologias baseadas na Internet torna o indivíduo mais eficiente \\
\hline & As pessoas me procuram para receber orientação de tecnologias on-line \\
\hline & Não preciso de ajuda de outras pessoas no uso de tecnologias on-line \\
\hline & $\begin{array}{l}\text { Estou entre os primeiros em meu círculo de amigos a aprender utilizar } \\
\text { tecnologias on-line }\end{array}$ \\
\hline & É muito complicado utilizar tecnologias baseadas na internet \\
\hline & $\begin{array}{l}\text { Sinto-me estranho quando tenho dificuldade em lidar com dispositivos on- } \\
\text { line e outras pessoas estão olhando para mim }\end{array}$ \\
\hline & As tecnologias on-line não são confiáveis \\
\hline & A Internet não é segura para transações monetárias \\
\hline & Existe falta de privacidade nas informações enviadas pela internet \\
\hline
\end{tabular}




\begin{tabular}{|l|l|}
\hline Constructo & Indicadores \\
\hline \multirow{4}{*}{$\begin{array}{l}\text { Persistência } \\
\text { Discente }\end{array}$} & Graduar-me em um curso a distância é importante para mim \\
\cline { 2 - 2 } & Estou confiante de que posso superar os obstáculos do curso EaD \\
\cline { 2 - 2 } & Certamente me matricularei no próximo semestre do meu curso EaD \\
\cline { 2 - 2 } & Concluirei o meu curso EaD não importa o quão difícil seja \\
\cline { 2 - 2 } & Eu não tenho possibilidade de continuar meus estudos no curso EaD \\
\cline { 2 - 2 } & Gostaria de abandonar meus estudos no curso EaD \\
\hline
\end{tabular}

Fonte: Elaboração própria (2017).

Recebido: 06/08/2018

Received: 08/06/2018

Aprovado: 08/02/2019

Approved: 02/08/2019 